\title{
A Survey of Humidified High-flow Nasal Cannula Usage in Indian Pediatric Intensive Care Units
}

\author{
Kalaimaran Sadasivam ${ }^{1}$, Bala Ramachandran ${ }^{2}$
}

Keywords: Humidified high-flow nasal cannula, Pediatric intensive care, Respiratory support, Ventilation.

Indian Journal of Critical Care Medicine (2020): 10.5005/jp-journals-10071-23628

Sir,

Humidified high-flow nasal cannula (HHFNC) has been increasingly used in the recent years. ${ }^{1-5}$ It is being used as an option for noninvasive respiratory support in children in many pediatric intensive care units (PICU). ${ }^{1-4}$ However, the current use of HHFNC in children is essentially based on individual hospital experience, as there are no existing national guidelines for the use of HHFNC in pediatric practice. Hence, our aim was to survey the Indian PICUs to identify the current practice of HHFNC usage.

We collected data by an online questionnaire (google survey) that assessed current HHFNC practice in Indian PICUs. The survey questions are listed in the Appendix and included information about the hospital, application of HHFNC, indications, and complications experienced. A total of 100 Indian PICUs were invited to participate. Participation was anonymous and voluntary. Ethical clearance was not required for the study. The statistical analysis was performed by SPSS version 24.

Of the 100 surveys sent out, we received responses from 72 PICUs. Sixty-nine (96\%) PICUs confirmed using HHFNC in their setting. The demographics of participating PICUs is summarized in Table 1. Of the 72 PICUs, 59 (82\%) used AIRVO ${ }^{\mathrm{TM}} 2$ system, and Optiflow $^{\mathrm{TM}}$ (Fisher and Paykel Healthcare, Auckland, New Zealand) was used by 27 (38\%). Clinical policies for the use of HHFNC were in place in 37 (51.4\%) PICUs.

Respiratory distress due to bronchiolitis (97.2\%) was the most common condition for using HHFNC, followed by pneumonia (87.5\%), and asthma (63.9\%). Sixty-eight (94.4\%) PICUs frequently applied HHFNC as respiratory support when weaning from invasive ventilation. Table 1 illustrates other indications and parameters for application of HHFNC. In addition, HHFNC was considered by PICUs as an alternative to continuous positive airway pressure (CPAP) (69.4\%) for escalating respiratory support from low flow oxygen (89\%) and weaning from CPAP or non-invasive ventilation (NIV) (54.2\%) (Table 1).

Nearly half (48\%) of the PICUs reported no complications or problems due to HHFNC. Mechanical issues (e.g., circuit condensation, system error, flow obstruction) were encountered by 26 (36\%) PICUs, whereas 25 (34\%) of 72 PICUs reported patient complications such as nasal trauma, epistaxis, and air leaks (Table 1). Most PICUs used either invasive ventilation (57\%) or NIV (43\%) when patients failed HHFNC.

\begin{abstract}
1,2Paediatric Intensive Care Unit, Kanchi Kamakoti CHILDS Trust Hospital, Chennai, Tamil Nadu, India

Corresponding Author: Kalaimaran Sadasivam, Paediatric Intensive Care Unit, Kanchi Kamakoti CHILDS Trust Hospital, Chennai, Tamil Nadu, India, Phone: +91 7358293442, e-mail: kalaimarans@gmail.com How to cite this article: Sadasivam K, Ramachandran B. A Survey of Humidified High-flow Nasal Cannula Usage in Indian Pediatric Intensive Care Units. Indian J Crit Care Med 2020;24(10):996-998.
\end{abstract}

Source of support: Nil

Conflict of interest: None

The main aim of the survey was to ascertain the use of HHFNC in Indian PICUs. As previously known, HHFNC is safe, well-tolerated, and higher oxygen concentration can be delivered. ${ }^{5} \mathrm{It}$ is also easy to use and setup when compared to NIV. ${ }^{4,5}$ Our survey has displayed that HHFNC is increasingly being used in Indian PICUs and is being considered for wider pediatric respiratory conditions, such as pneumonia, asthma, or as a respiratory support in patients being weaned from invasive or noninvasive ventilation. This observation is consistent with other publications on use of HHFNC. ${ }^{4-6}$ Although most of the PICUs in our survey have used HHFNC for children with primary respiratory problems, some $(40.3 \%)$ reported using it for children with underlying cardiac diseases or post cardiac surgery (Table 1), and a few (9\%) have used for other conditions such as neuromuscular disorders, septic shock, and post-endoscopic procedures.

The most common benefits reported by PICUs for using HHFNC were the ease of use and improved tolerance as well as to reduce nasal trauma, which is similar to the other available surveys. ${ }^{2,4}$ Complications associated with HHFNC are most frequently due to tubing rainout or circuit condensation that may be linked to the cooling of the HHFNC gas below its dew point. ${ }^{2,5}$ Consistent with this, almost a third of our PICUs stated circuit condensation as a main problem (Table 1). The number of patient injury in our study has also been similar to the previous publications ${ }^{4-6}$ (Table 1). Only 11 PICUs encountered side effects of nasal trauma, including bleeding of the nasal mucosa, and 14 PICUs reported air leak complications such as pneumothorax. The rate of patient-related injury due to HHFNC

(-) The Author(s). 2020 Open Access This article is distributed under the terms of the Creative Commons Attribution 4.0 International License (https://creativecommons. org/licenses/by-nc/4.0/), which permits unrestricted use, distribution, and non-commercial reproduction in any medium, provided you give appropriate credit to the original author(s) and the source, provide a link to the Creative Commons license, and indicate if changes were made. The Creative Commons Public Domain Dedication waiver (http://creativecommons.org/publicdomain/zero/1.0/) applies to the data made available in this article, unless otherwise stated. 
Table 1: Respondent demographics and survey responses

\begin{tabular}{|c|c|c|}
\hline HHFNC usage & $n$ & (\%) \\
\hline \multicolumn{3}{|l|}{ Type of hospital } \\
\hline Government & 7 & $(9.7)$ \\
\hline Private & 56 & $(77.8)$ \\
\hline Private non-profit & 9 & $(12.5)$ \\
\hline \multicolumn{3}{|l|}{ Type of PICU } \\
\hline Pediatric medical & 18 & $(25.0)$ \\
\hline Neonatal and pediatric medical & 38 & $(52.8)$ \\
\hline Medical and Surgical & 42 & $(58.3)$ \\
\hline Cardiac & 14 & $(19.4)$ \\
\hline Adult and pediatric medical and surgical & 4 & $(5.6)$ \\
\hline \multicolumn{3}{|l|}{ No of PICU beds } \\
\hline$<6$ & 6 & $(8.3)$ \\
\hline $6-12$ & 43 & $(59.7)$ \\
\hline$>12$ & 23 & (31.9) \\
\hline \multicolumn{3}{|l|}{ Years since PICU functional } \\
\hline$<5$ years & 20 & $(27.8)$ \\
\hline$>5$ years & 52 & $(72.2)$ \\
\hline \multicolumn{3}{|l|}{ Where HHFNC is used } \\
\hline PICU & 71 & $(100.0)$ \\
\hline High dependency unit (HDU) & 38 & $(53.5)$ \\
\hline Wards & 10 & $(14.1)$ \\
\hline Emergency rooms & 8 & $(11.3)$ \\
\hline Operation theaters & 1 & $(1.4)$ \\
\hline \multicolumn{3}{|l|}{ Years of using HHFNC } \\
\hline$<2$ years & 18 & $(25.0)$ \\
\hline $2-4$ years & 35 & $(48.6)$ \\
\hline$>4$ years & 19 & $(26.4)$ \\
\hline \multicolumn{3}{|l|}{ Indications for using HHFNC } \\
\hline Bronchiolitis & 70 & $(97.2)$ \\
\hline Pneumonia & 63 & $(87.5)$ \\
\hline Post extubation & 68 & $(94.4)$ \\
\hline Asthma & 46 & $(63.9)$ \\
\hline Alternative to CPAP & 50 & $(69.4)$ \\
\hline $\begin{array}{l}\text { Escalating respiratory support from low } \\
\text { flow oxygen }\end{array}$ & 64 & $(89)$ \\
\hline Wean from CPAP or NIV & 39 & $(54.2)$ \\
\hline Wheezy lower respiratory infections (LRI) & 54 & (75) \\
\hline Post-operative cardiac & 29 & $(40.3)$ \\
\hline Stridor & 34 & $(47.2)$ \\
\hline Other* & 7 & (9) \\
\hline \multicolumn{3}{|l|}{ Complications due to HHFNC } \\
\hline No complications & 35 & $(48)$ \\
\hline Nasal trauma & 9 & $(12)$ \\
\hline Air leak syndorme & 14 & (19) \\
\hline Epistaxis & 2 & (3) \\
\hline Mechanical issues with HHFNC & 26 & (36) \\
\hline
\end{tabular}

*Other conditions like neuromuscular disorders, septic shock and post endoscopic procedures

HHFNC, humidified high-flow nasal cannula; PICU, pediatric intensive care units is comparatively less than NIV, ${ }^{4}$ and due to the ease of usage, it is increasingly becoming one of the preferred options for respiratory support in children., ${ }^{4,5}$

The main issue highlighted in our survey is the process of sterilizing and reusing the HHFNC circuit. Although the manufacturer of HHFNC does not recommend reusing the circuit, many (60\%) PICUs in our survey were reusing the circuit. This could be attributed to the cost of HHFNC circuit which many patients and families may find challenging; hence, the hospitals choosing to sterilize and reuse them. The majority (79.2\%) of our PICUs emphasized the need for more elaborate studies to validate the use of HHFNC, and nearly all (96\%) would like to participate and contribute in clinical trials.

Our study is the first national survey to review the HHFNC usage in Indian PICUs as well as highlighting the prevalent issues related to its use. Our results suggest that despite limited evidence on efficacy of HHFNC and the limitations of cost, it is still widely used and is considered safe.

\section{Acknowledgments}

We are thankful to all the PICUs and respondents for completing the survey and for their useful comments.

\section{References}

1. Miller AG, Gentle MA, Tyler LM, Napolitano N. High-flow nasal cannula in pediatric patients: a survey of clinical practice. Respir Care 2018;63(7):894-899. DOI: 10.4187/respcare.05961.

2. Hough JL, Shearman AD, Jardine LA, Davies MW. Humidified high flow nasal cannulae: current practice in Australasian nurseries, a survey. J Paediatr Child Health 2012;48(2):106-113. DOI: 10.1111/j.14401754.2011.02070.x.

3. Hosheh O, Edwards CT, Ramnarayan P. A nationwide survey on the use of heated humidified high flow oxygen therapy on the paediatric wards in the UK: current practice and research priorities. BMC Pediatr 2020;20(1):1-9. DOI: 10.1186/s12887-020-1998-1.

4. Schmid F, Olbertz DM, Ballmann M. The use of high-flow nasal cannula (HFNC) as respiratory support in neonatal and pediatric intensive care units in Germany - a nationwide survey. Respir Med [Internet] 2017;131:210-214. DOI: 10.1016/j.rmed.2017.08.027.

5. Mikalsen IB, Davis P, Øymar K. High flow nasal cannula in children: a literature review. Scand J Trauma Resusc Emerg Med [Internet] 2016;24(1). DOI: 10.1186/s13049-016-0278-4.

6. Baudin F, Gagnon S, Crulli B, Proulx F, Jouvet P, Emeriaud G. Modalities and complications associated with the use of high-flow nasal cannula: experience in a pediatric ICU. Respir Care 2016;61(10):1305-1310. DOI: 10.4187/respcare.04452. 


\section{Appendix}

\begin{tabular}{|c|c|}
\hline \multicolumn{2}{|c|}{ Survey questions: } \\
\hline 1 & Name of hospital and city/town \\
\hline 2 & What is your position in the hospital? \\
\hline 3 & What kind of hospital? \\
\hline 4 & Type of PICU. \\
\hline 5 & Number of PICU beds \\
\hline 6 & How long has the PICU been operational? \\
\hline 7 & Do you currently use NIV? \\
\hline 8 & Do you use HFNC? \\
\hline 9 & How long has your unit been using HFNC \\
\hline 10 & Which disease processes/situations is HFNC used. \\
\hline 11 & When do you consider HFNC? \\
\hline 12 & If HFNC fails - Which one of the following do you consider as the 1st option? \\
\hline 13 & Do you have written unit guidelines/protocols for initiating, evaluating and stopping HFNC? \\
\hline 14 & Where do you use HFNC? Tick all that are applicable \\
\hline 15 & Which brand of HFNC do you use? Tick all that are applicable \\
\hline 16 & Do you reuse HFNC circuits? \\
\hline 17 & How do you sterilise HFNC circuits? \\
\hline 18 & What problems with HFNC have you encountered on your unit? \\
\hline 19 & Do you include HFNC as NIV for bed state/audit/data purposes? \\
\hline 20 & Is your unit currently involved in clinical research into HFNC? \\
\hline 21 & Would your unit consider joining clinical trials of HFNC? \\
\hline
\end{tabular}

